\title{
CUERPO Y POLÍTICA
}





\title{
El cuerpo fascista recuperado: la exploración de la masculinidad en Fight Club
}

\author{
The Fascist Body Reconfigured: Masculinity \\ explored in Fight Club
}

LEONOR ACOSTA BUSTAMANTE*

\begin{abstract}
Resumen: En 1999 los debates sobre la masculinidad en crisis produjeron en Estados Unidos un volumen importante de literatura crítica y posicionó en bandos contrarios a los estudiosos que provenían de las proclamas feministas y los que fundaron el movimiento mitopoético de los hombres, enfrentados a las máximas de los Estudios de Género. El estreno de Fight Club provocó una especie de catarsis en ese escenario de confrontación y abordó la regeneración de la masculinidad hegemónica, localizándola en la vuelta a la violencia primitiva como esencia masculina. Entroncando así la narración entretejida con esos discursos, la apuesta de David Fincher, y de Chuk Palahniuk en la novela homónima de la que parte la película, aboga por detectar los peligros de entender lo masculino como esencialmente vinculado al dolor físico y a la agresividad del combate, provocando la identificación de este proceso con la conformación de los cuerpos políticos del fascismo. Palabras clave: Masculinidad, cuerpo masculino, violencia, Movimiento Mitopoético, fascismo, Fight Club.
\end{abstract}

\begin{abstract}
In 1999 the debates around the crisis of masculinity produced in the United States of America a great amount of criticism and situated in opposition the scholars coming from the feminist environment and those who founded the Mythopoetic Movement of Men, confronted to the assumptions of the discipline of Gender Studies. The release of Fight Club provoked a catharsis in that scenario of confrontation and addressed the regeneration of hegemonic masculinity, locating it in a regression towards primitive violence conceptualized as male essence. By assembling the narrative with these discourses, David Fincher's proposal (as well as Chuk Palahniuk's in the novel originating the film), intends to diagnose the perils of understanding the masculine as essentially linked to physical pain and the aggressive combat, provoking the identification of this process with the formation of the political bodies of fascism.
\end{abstract}

Keywords: Masculinity, male body, violence, Mythopoetic movement, fascism, Fight Club.

Fecha de recepción: 12/06/2016. Fecha de aceptación: 06/09/2016.

* Universidad de Cádiz. Profesora de Estudios Culturales en el Departamento de Filología Francesa e Inglesa (leonor.acosta@uca.es). Las líneas de investigación vigentes en las que trabaja son: "Masculinidades y Estudios de Género", "Estudios Culturales" y "Estudios Fílmicos". Entre las publicaciones recientes se encuentran los siguientes capítulos de libro: "Un libro es un arma cargada en la casa de al lado": El fin de la cultura y el apocalipsis en Fahrenheit 451”, en El arte a juicio (Eds. María Jesús Ruiz y Luis Ramón Ruiz). Valencia: Tirant Lo Blanch, 2009; y “Aproximación a los Estudios de Masculinidades: Teoría y aplicaciones”, en Género y ciencias sociales. Arqueologías y cartografias de fronteras. (Eds. Fernanda Pattaro Amaral y Ma Nohemí González Martínez). Barranquilla, Colombia: Ediciones Universidad Simón Bolívar, 2015. En prensa se encuentra el capítulo de libro en colaboración titulado "Alternative Sexualities in Virtual Communities: Aspects of LGTB Participation on Social Networking Sites", en Identity, Sexuality, and Relationships among Emerging Adults in the Digital Age (Ed. Michelle Wright). Hershey, Pennsylvania: IGI Global. 
En 1996 George L. Mosse dedicaba un capítulo de su libro The Image of Man. The Creation of Modern Masculinity al concepto del hombre fascista. En un recorrido por las ideas acerca de la masculinidad desde su aparición en el pensamiento occidental como parte del ideario ilustrado, Mosse demuestra cómo los estereotipos creados para definir el 'ser hombre' devienen en un fenómeno histórico que condiciona y afecta las diferentes maneras de construir las sociedades modernas. En el análisis que propone para el capítulo dedicado a las relaciones entre el cuerpo político del fascismo de los años 30 y el cuerpo masculino, Mosse localiza este estereotipo como consecuencia directa de la primera guerra mundial en el entorno del nacionalismo italiano ayudado en el terreno de las artes por el movimiento futurista italiano. En este conglomerado de ideas, la masculinidad recoge una red conceptual que contiene parte de las construcciones del pasado clásico, como la identificación entre salud mental y salud física -mens sana in corpore sano-, con añadidos más entroncados con la conciencia política nacionalista que conectaba la salud del cuerpo y la mente con el entusiasmo inescapable por la lealtad a la patria, entendida como una comunidad de hombres leales a un proyecto ideológico común y a una bandera.

La ideología nacionalista se construye en este periodo de posguerra olvidando sus conexiones de revitalización de una tradición propia para mirar a un futuro celebratorio de progreso, tecnología y preponderancia de la nación (italiana) por encima de las otras naciones de la tierra. En este sentido la nueva masculinidad promulgada por los fascismos suponía un acercamiento espiritual revolucionario por el que el individuo debía poner su cuerpo y su mente al servicio de una causa trascendental mayor en beneficio de una élite llamada a un estadio más allá de lo ordinario. En la creación de esta identidad masculina las ideas fascistas se dejaron condicionar y bebieron íntimamente de los mandamientos del Futurismo, que incidían en desgranar las características propias del Hombre Nuevo, cuyas bases principales eran la abolición de todo lo antiguo y la adopción eufórica de la modernidad y todo lo que ella proponía. De esta manera las propuestas futuristas apuntaban a un individuo que no estuviese anclado por el peso del pasado y que pudiera proclamar la gloria de Italia en el uso revolucionario de la confrontación y el combate enérgico para poner su sacrificio y su sufrimiento al servicio de la patria. El hombre se convierte en élite por medio de su afán de lucha, su actividad bélica y su desprecio por todo lo burgués, todo lo femenino y todo lo que debilita su naturaleza viril.

En la recopilación de 1968 publicada en español por la Editorial del Cotal en Barcelona con el título Manifiestos y textos futuristas de Filippo Tommaso Marinetti, se recoge una serie de contribuciones del fundador del Futurismo como glosas del primer Manifiesto Futurista publicado en Le Figaro en 1909. En ellos Marinetti abunda en estas proclamas que parten de directrices para la revolución estética pero que fomentaron la ideología fascista en la Italia de principios de siglo XX. El amor a la guerra y la celebración de la violencia tenían en sus escritos una vinculación clara con la necesaria modificación de los cuerpos masculinos, en un divorcio completo de los lazos previos que los unían a la feminidad y al sentimentalismo:

¿Quién puede afirmar que un hombre fuerte no respira mucho mejor, no come mucho mejor, no duerme mucho mejor que de costumbre después de haber abofeteado y derrumbado a su enemigo? ¿Quién puede afirmar que la palabra hombre y la palabra luchador no son sinónimas? (Marinetti, 1968, 62) 
Marinetti se creaba para sí mismo una imagen de liderazgo encarnada en la metáfora del Nuevo Hombre italiano para alimentar el orgullo nacionalista basado en la bravura y el odio instaurados como esencia en cuerpos masculinos en constante combate. Esta nueva idea de la masculinidad se fue alimentando progresivamente por medio, en primer lugar, de la experiencia de los soldados en la segunda guerra mundial, y más tarde por medio de las proclamas fascistas que incidían en la identificación entre cuerpo político del estado y el cuerpo masculino vigoroso y viril. Más aún, la experiencia bélica celebraba la camaradería masculina como parte de esa virilidad apartada del contacto con lo femenino, que, además, provocó una serie de directrices para la consolidación del fascismo alrededor del interés por el cuerpo de los hombres como emblema nacional: el uso constante de los uniformes militares, las marchas, el énfasis puesto en el deporte y el ejercicio físico se convirtieron en una regularización de la batalla como experiencia vital incluso sin estar viviendo en medio de un conflicto bélico e implicaba una pureza moral necesaria para la glorificación del estado.

The overriding importance of physical exercise in Italy, was not so much centered on the symbolic importance of the beautiful body as, above all, on its use to instill discipline and a sense of order, and proper comportment was considered essential. That physical exercise furthered comradeship was considered vital as well; here a real camaraderie was said to exist (as it had in the war), one that knew neither class nor caste... This was no small matter, remembering that the ideal of male comradeship, as far as fascism and National Socialism were concerned, provided the foundation of the state. (Mosse, 1996, 161) ${ }^{1}$

Desde esta perspectiva, el cuerpo masculino desnudo, con caracteres claramente ligados a las figuras escultóricas de la época griega y romana, generaba significados de salud mental y pureza espiritual, a la vez que enlazaba el futuro del fascismo italiano con el pasado imperial. La figura del hombre desnudo con tales elementos corporales (belleza clásica, armonía entre sus partes, musculatura y pose) aglutinó los valores positivos de moralidad, patriotismo guerrero, salud y fuerza física, violencia y valentía en la sangre, para conceptualizar a la nación fascista.

Tras el declive de los mensajes totalitarios, y después de la derrota del nacionalsocialismo alemán tras la segunda guerra mundial, la idea del patriotismo seduce al gobierno estadounidense, que recupera la idea de la identidad norteamericana como la bandera de la globalización, justificada teóricamente con la idea de expandir la democracia, la ideología burguesa estadounidense y el capitalismo consumista (Cuordileone, 2005). Estos valores, propios de la Guerra Fría y creados en oposición a la ideología fascista, se enumeran como la única alternativa ante la invasión comunista y construyen al individuo formalmente como un sujeto dado a las emociones: la nación no debe construirse si no es por medio de familias

1 "La importancia primordial del ejercicio físico en Italia no se centraba tanto en la importancia simbólica de un cuerpo hermoso, sino en su uso para inculcar la disciplina y el sentido del orden, considerándose esencial un comportamiento apropiado. También se consideraba vital el ejercicio físico como fomento de la camaradería, una camaradería real (tal como la que existía en la guerra) que pasara por encima de clases y de castas.... Esto no es una cuestión baladí, si se recuerda que el ideal de camaradería masculina, hasta el punto en que concernía al fascismo y al nacional-socialismo, proveía el fundamento para el estado". (traducción de la autora). 
nucleares unidas por el amor, dando así carta abierta al patriarcado a instalarse contundentemente en la vida de los estadounidenses. Este 'debilitamiento' de la masculinidad, que, aunque patriarcal, se aleja de la bravura patriótica y fascista de la primera mitad del siglo $\mathrm{XX}$, es el caldo de cultivo para que años más tardes se deje afectar por los dictados del feminismo emergente y de otros activismos sociales, a saber, los que conformaron la época de los Derechos Civiles en Estados Unidos (Ling \& Monteith, 1999).

En este continuum histórico, que produce paradójicamente una discontinidad en la masculinidad creada diacrónicamente para adaptarse a los tiempos de posguerra, los estereotipos culturales de la masculinidad hegemónica de décadas anteriores se 'feminizan' y se orientan hacia un hombre emocionalmente capaz de afrontar los embates de la contracultura, originando el fenómeno de la crisis de la masculinidad que se torna en cuestión casi de estado entre las décadas de 1980 y 1990 (Carrigan, Connell \& Lee, 1985; Connell, 1995; Kimmel, 1996). Con la entrada de las teorías feministas acerca del género como constructo social, la masculinidad se convierte en una de las construcciones debatidas en ese foro, reforzada por un siglo de estudios antropológicos y luego sociológicos acerca de la conceptualización de lo masculino frente a lo femenino (Ward 1883; Mead 1935). Tal como lo explica Henry A. Giroux,

A los hombres blancos heterosexuales de Estados Unidos no les ha ido muy bien en la década de los noventa. No sólo los han atacado las feministas, los gays y las lesbianas y distintos grupos subalternos a partir de una variedad de ofensas ideológicas y materiales, también han tenido que soportar la reescritura del significado de la masculinidad... El destino manifiesto de la masculinidad, con su severa imagen de dureza y de hombría, ha sido perturbado y la cerrazón de su capacidad de reflexión profundamente cuestionada. (Giroux, 2003, 286)

Habiendo encarnado al sujeto de los humanismos y al agente de la nación estadounidense como emblema de la modernidad, el hombre heterosexual blanco se convierte en el objeto más evidente de la crisis identitaria del periodo pos-sesentista. No es que la masculinidad pierda su estatus de privilegio al entrar en la discusión feminista y pos-feminista, es que esta masculinidad hegemónica pierde el propio sentido del ser, en un régimen económico neoliberal (el tardo-capitalismo posmodernista) (Jameson, 1991) que lo descoloca y lo transforma en un objeto para el espectáculo del consumo. De una economía de base manufacturada en la que los hombres eran productores de bienes, la sociedad de finales del siglo XX pasó a basar su economía en las corporaciones multinacionales, los mercados globalizados y los movimientos deslocalizados del capital. Esta última fase del capitalismo también reforzó en esos mismos años la profunda alienación del sujeto actante del primer capitalismo -competitivo y productivo- trasladándolo a un territorio inhóspito e incomprensible, mientras las minorías sociales (los 'otros' géneros, razas, clases sociales, etnias, etc.) tomaban las riendas de los discursos identitarios y se hacían fuertes ante dicha hegemonía.

Un producto directo de este fenómeno es la aparición y consolidación de los Estudios de Masculinidades que arrancan desde el contexto teórico sostenido por los Estudios de Género, para producir desde la década de los 80 una ingente literatura crítica acerca de las tipologías masculinas, la construcción social y cultural de la masculinidad hegemónica y las diferentes representaciones de las masculinidades en la cultura popular, abogando desde 
un principio por una diversificación en los acercamientos y las disciplinas que acogen este nuevo corpus (Adams \& Savran 2002; Reeser 2010; Whitehead \& Barret 2001). En torno a esta nueva disciplina, que bebe de fuentes de la antropología, la sociología, la teoría literaria, los estudios culturales, las ciencias sociales, y, por supuesto, los estudios de género, se reunieron versiones diversas sobre la masculinidad con diferentes desarrollos ideológicos.

Entre estos desarrollos diversos de estudio y análisis de las masculinidades es destacable, por las consecuencias culturales que provocó en la década de los 90, el enfrentamiento entre los sociólogos culturalistas entroncados con la línea de los posfeminismos y ciertas asociaciones conservadoras alineadas en el movimiento llamado 'mitopoético' proveniente de los "Estudios de Hombres". ${ }^{2}$ Como su propio nombre indica, el movimiento mitopoético reclamaba una regeneración de los hombres por medio de la revitalización de los mitos del pasado pre-industrial con el objeto de recobrar la potencia de la masculinidad primitiva. ${ }^{3}$ Los hombres alineados bajo este paraguas político y social demandan una vuelta a los roles de protectores, proveedores y padres. Tomando en consideración la idea de que la debilidad masculina proviene directamente del pensamiento ilustrado y de la modernidad, los hombres mitopoéticos abrazan el primitivismo como fuente de energía conceptual, extendiendo esta idea a través de un asociacionismo activista gestionado en grupos de autoayuda para hombres en busca de su virilidad perdida. En el ritual programado por el Proyecto Mitopoético la consigna es recuperar el poder masculino en comunidades homosociales creando una 'hermandad' de hombres desahuciados por la sociedad capitalista y separados de su verdadera naturaleza masculina por el contacto constante con el entorno femenino. Este activismo social, que engendró un programa de entrenamiento para conseguir los fines del movimiento fundado con el nombre de "La Aventura Formativa del Nuevo Guerrero" ("New Warrior Training Adventure"), comparte algunos de los fundamentos de los proyectos fascistas de principios de siglo, alimentado simultáneamente de cierta carga sentimental (homosocial) que lo complementa. El desarrollo de esta 'aventura' (llamada también "el viaje del héroe") se explica de esta manera:

Desde el viernes por la tarde hasta el domingo (con comida, espacios para el descanso y tiempo para dormir) deberás realizar una serie de actividades -todas diseñadas con un objetivo en mente- para ayudarte paso a paso a través de un entrenamiento para encontrar el sentido del ser en una comunidad de hombres. Algunas de las actividades son individuales pero la mayoría se realizan dentro del grupo. Son ejercicios físicos pero también hay espacio para la escritura y visionados de materiales. Al volver a casa el domingo serás un hombre nuevo, habrás concluido el viaje del héroe. ${ }^{4}$

2 Los Estudios de Hombres (Men's Studies) se diferenciaban de los Estudios Masculinistas (Masculinity Studies) en su propia denominación, dado que la selección de la palabra/concepto "hombre" (men) proponía una recuperación de la identidad masculina como una esencia y no como un constructo cultural y social (más evidente en el uso de la palabra 'masculinidad').

3 Es algo convenido por la crítica que este movimiento tiene su origen en el libro Iron John, publicado en 1990 por el poeta Robert Bly, donde el autor conecta esa fuerza primitiva con las figuras mitológicas de Zeus, el rey Arturo, y el héroe de cuento Juan Sin Miedo.

4 Traducción de la autora. Tomado de la página web del proyecto enmarcado por el texto: "The Mankind Project International. Changing the world one man at a time since 1985: We were scared too, you'll get through it" (El proyecto internacional de los hombres. Cambiando el mundo de hombre a hombre desde 1985. Nosotros también estábamos asustados. Lo conseguirás"). http://mankindproject.org/ 
El estreno de la película Fight Club en 1999, dirigida por el afamado David Fincher ${ }^{5}$ y basada en la novela de Chuck Palahniuk (1996), produjo un voluminoso número de respuestas por parte de la crítica que posicionó a este texto fílmico en un espacio de debate muy convenientemente conectado con las discusiones finiseculares sobre la masculinidad y su crisis. La compleja y polémica presentación del tema -la vuelta a la violencia como esencia del hombre- nutrió la expectación mediática en dos niveles de recepción: por una parte, el estreno en el 56 Festival de Cine de Venecia en septiembre de 1999 permitió la categorización de la película de Fincher como cine de culto ${ }^{6}$; por otra parte, el éxito que obtuvo en Europa produjo un éxito de taquilla impensable al estrenarse en Estados Unidos un mes más tarde. Esta doble vía de distribución hizo de Fight Club un fenómeno cultural de una magnitud nunca imaginada en sus comienzos. Y posiblemente la causa sea el haber trastocado los límites del mercado hollywoodiense para colocar en la pantalla reflexiones problemáticas sobre el individuo perdido en una sociedad hostil y enajenante para abordar la cuestión masculina con la ansiedad típica del cambio de centuria. Las interpretaciones de Brad Pitt en el papel de Tyler Durden y de Edward Norton como el protagonista masculino, acompañado de Helena Boham Carter como Marla Singer, aportan un sinfín de matices interesantes y llenos de significados para un imaginario colectivo aleccionado en constante adiestramiento de roles patriarcales de género. Es más. la atención monumental de la crítica académica al complejo texto previamente inventado por Palahniuk posiblemente siga siendo un indicador suficientemente significativo para considerar esta película como un tejido rico en interpretaciones sobre la problemática naturaleza de los hombres, sobre la modernidad de Estados Unidos, sobre la sociedad occidental contemporánea, sobre la deshumanización provocada por el capitalismo, o sobre el fin de la historia.

Retomando un tópico proveniente del Romanticismo alemán, el texto fílmico utiliza la figura del doble para introducir en él el debate sobre lo masculino. El protagonista principal, un personaje llamado Jack, ${ }^{7}$ se presenta en las primeras escenas como un hombre acomodado en un estado de debilidad vital y a una existencia basada en la aceptación del consumismo como única vía de afirmación ante la alienación provocada por el trabajo de peritaje en una compañía de seguros y una vida privada caracterizada por un insomnio constante. En uno de sus viajes en avión encuentra (se proyecta en) un alter-ego, Tyler Durden, que le inicia en un camino hacia experiencias antisistema y que le abre los ojos a una nueva masculinidad basada en la experiencia de la violencia y la lucha. El tópico mitopoético se traslada así a la pantalla en monólogos de Tyler que entrena al protagonista

5 Dice Devin Orgeron que David Fincher es el último director de películas sobre ideas que se posicionan contra el sistema de finales del siglo XX en Hollywood (Orgeron 2002, 154-155). Utilizando estructuras narrativas populares enmarcadas en el género fantástico, Fincher es capaz de insertar en ellas una crítica social y política sobre la alienación y la deshumanización del individuo contemporáneo. En 1999 Fincher había conseguido ese lugar en la historia del cine estadounidense por sus tres éxitos anteriores: Alien 3 (1992), Se7en (1995) y The Game (1997).

6 Los productores ejecutivos de Hollywood habían interpuesto algunos obstáculos para su estreno por no encontrar, precisamente, un espacio dentro de las estructuras del mercado en Estados Unidos. El gran problema era no poder promocionar la película como, en principio, la entendieron: como una película de boxeo. La polémica de cómo se representaban a los hombres dejaba sin posibilidades de incluirla en estas fórmulas masculinas.

7 Aunque nunca nadie se dirige a él lo llama por su nombre a lo largo de la película. Es precisamente el propio personaje el que se refiere a sí mismo en letanías en las que reflexiona sobre sí y expresa esas reflexiones en tercera persona. 
a 'ser hombre', apuntando a las causas de la alienación en el mismo sentido en el que lo hacía Bly y sus seguidores. "Somos una sociedad de hombres criados por mujeres, no sé si una mujer es la solución".

Aparte de caracterizarse como consumidor adicto a IKEA, ${ }^{8}$ el protagonista también se presenta como un individuo insomne al que solo le hacen dormir las sensaciones de comunidad que experimenta en el grupo de terapia para hombres discapacitados (hombres con cáncer de testículos). $\mathrm{Y}$ es en esta terapia donde se produce el encuentro con dos de los personajes más relevantes de la narrativa. En primer lugar, Marla Singer, la única mujer representada en el texto, es un personaje muy alejado de los estereotipos femeninos: alienada como el protagonista, alternativa en su modo de proceder y en sus actividades, Marla es un 'outsider', una no-persona en el mundo consumista habitado por el personaje principal, cuya presencia le molesta y perturba en su búsqueda de la estabilidad emocional. Sin embargo, el contacto con Bob, el segundo personaje fundamental en esta exploración narrativa, provoca en él la tranquilidad y el sosiego cuando este hombre le abraza y le permite llorar en sus pechos, producto de la toma masiva de anabolizantes y de la castración quirúrgica de sus testículos por culpa del cáncer.

Marla y Bob son los ejes principales que utiliza la película para establecer el conflicto entre la masculinidad y la feminidad encarnado de forma visible en los cuerpos de cada uno: cuerpos híbridos que trastocan la apariencia tradicional de los sexos dado que ninguno de los dos cumplen con los códigos de construcción del cuerpo insertada en la codificación del género. Desde esta perspectiva los dos personajes que rodean al protagonista forman un eje común para debatir sobre cuestiones de choque contra lo convencional que ambos desmantelan para que el protagonista se pierda entre los pechos maternales de Bob y rechace en cambio a Marla porque abomina el contacto con una mujer biológica.

No obstante es la relación obsesiva con su alter-ego lo que proporciona a la película todo el peso específico sobre las cuestiones de qué desarrollo puede tener la revitalización de la hombría siguiendo las directrices mitopoéticas. Y aquí la narrativa provee al espectador de múltiples mensajes no lineales ni definitivos. Por un lado, el encuentro constante entre los dos estereotipos (el 'debil' burgués y el 'fuerte' antisistema) no permite fácilmente desdeñar al que enarbola las ideas antifeministas en favor de la violencia y la fuerza física, fundamentalmente porque es Brad Pitt el actor que interpreta a Tyler, $y$, porque en el viaje propuesto el protagonista va ganando confianza y poder sobre los que antes lo maltrataban. Es algunas veces desagradable relacionar esta fortaleza con los cuerpos heridos de los hombres peleando hasta el último grado de resistencia y con los signos de ese maltrato en el centro de la pantalla, pero de alguna manera, el espectador empatiza con la lucha como, tal como expresa Asbjorn Gronstad, una práctica y un signo que separa el ser de la nada. (Gronstad, 2003, 1).

La fundación del Club de la Lucha como asociación de hombres con un solo objeto, el de luchar para recobrar el sentido de la hombría, contiene todos los elementos para relacionarlo por oposición a los grupos de terapia introducidos al principio de la narrativa, solo que el

8 IKEA simboliza aquí un modelo identitario clave para entender al tipo de masculinidad que representa el protagonista: hombre joven, blanco y heterosexual pero a la vez disidente de la cultura media norteamericana, consumista de productos europeos y encarnando al soltero por necesidad, dado la problemática de conseguir entablar relaciones de cualquier tipo. 
decálogo establecido por Tyler Durden como eje de las actividades del club propone unas reglas que se alejan completamente del sentimentalismo del asociacionismo terapéutico y emocional al uso:

1. No hablar del Club de la Lucha

2. No hablar del Club de la Lucha

3. Si un luchador pronuncia la palabra DETENTE, la lucha acaba.

4. Solo dos hombres por cada combate.

5. Solo un combate cada vez.

6. Sin camisa y sin zapatos.

7. El combate dura lo que tenga que durar.

8. Si esta es tu primera noche, entonces tienes que luchar.

Desde este momento fílmico, se van editando secuencias de combates nocturnos yuxtapuestas a escenas en las que los personajes con signos de la pelea viven sus vidas habituales y en las que se van expresando señales de camaradería masculina. Participar en el combate, sufrir los golpes, sangrar se convierten así en elementos de una adicción que saca a los hombres de la hostilidad del mundo tecnológico y consumista para el encuentro con sus cuerpos 'primitivos' en la lucha. Y, aunque en esta fase todavía el espectador no sabe qué está bien y qué no, es a partir de la mitad del metraje cuando esta violencia se torna mucho más peligrosa y cuando las proclamas mitopoéticas se tornan en mensajes propios del totalitarismo político. Es el momento en que Tyler decide crear el Proyecto Manheimm, una agrupación con tintes fascistas que adora a su líder y le sigue hasta la muerte. Estos hombres, ${ }^{9}$ que viven juntos en Paper Street, y que pasan por rituales de iniciación para poder ser aceptados como miembros, que se entrenan en actividades terroristas (como trabajadores de la Paper Street Soap Company) y que se golpean todas las noches, transforman la narrativa y el espectador empieza a ver el peligro social de un grupo de hombres educados en el decálogo de Tyler. El Proyecto Manheimm va evolucionando a lo largo de la película hacia un anarquismo terrorista desde acciones simbólicas hasta el clímax final de organizar una actuación del grupo para demoler los edificios emblemáticos del capitalismo estadounidense simbolizado en la ciudad de Nueva York.

El sueño del guerrero interpretado por Brad Pitt que imagina una civilización preindustrial y primitiva de hombres poderosos es al final de la película el elemento más problemático y así se recoge en la escena final: el protagonista atado en la planta alta del edificio Parker-Morris con una pistola en la boca sostenida por Tyler, que tiene la intención de obligarlo a ser testigo de la destrucción de la Nueva York capitalista. Es entonces cuando la pistola se dispara, Tyler desaparece, pero el protagonista permanece vivo, de pie, de espaldas, cogido de la mano de Marla, mientras tras los cristales de la enorme ventana los rascacielos neoyorquinos arden en medio de la noche.

9 La apariencia de estos cuerpos refuerzan la identificación del Proyecto Manheimm con las milicias fascistas: los uniformes negros, el corte de pelo, el militarismo de sus gestos corporales enseguida generan inferencias significativas con el imaginario popular sobre el nacionalsocialismo alemán y el fascismo italiano de primera mitad del siglo XX. 
De ninguna manera ofrece este final ninguna sensación de tranquilidad en este escena apocalíptica, más bien lo contrario: en este final abierto, se reconoce a Tyler como una proyección mental del protagonista, y se entiende la narrativa como un viaje iniciático de este hombre desde la alienación consumista a través de unas sendas muy problemáticas, en las que ha aprendido a sufrir (no emocionalmente sino físicamente), a resistir el dolor hasta el máximo, a dejar atrás el peso del pasado, y a reconsiderar a Marla como una acompañante, mientras los dos se convierten en espectadores de la destrucción de "cada fibra de la historia", como si de los nuevos Adán y Eva se tratara.

En suma, Palahniuk primero y Fincher después parecen proponer una reflexión acerca de esa vuelta a lo primitivo que proclamaban (y siguen proclamando) los asociacionismos derivados del movimiento mitopoético. Sobreentendiendo el componente fantástico del doble psicológico, Jack se abandona a una experiencia comunitaria homosocial que puede llegar a provocar un estado de destrucción social y de enajenación de los hombres perdidos no ya en el mundo capitalista y consumista contemporáneo sino en un entorno alimentado solo por los instintos más agresivos. No parece la de Fincher, desde esta perspectiva, una propuesta conservadora (Giroux, 2003) que refuerza la celebración de la violencia como esencia masculina, sino más bien una exploración de ideas que reclaman finalmente la abolición del hombre unidimensional tan repetidamente recreado en el cine de Hollywood (Gronstad, 2003). Sin embargo, hay matices dentro de dicha exploración que apuntan más allá y la incorporan al debate de los Estudios de Masculinidades contemporáneo al estreno de la película y que tienen como objeto responder a las premisas del Proyecto Mitopoético de los hombres, erigido como entrenamiento para la regeneración de la masculinidad hegemónica que, según se desarrolla en la trama, puede degenerar en la experiencia masculina de los fascismos europeos del pasado.

\section{Obras citadas}

Adams, R. \& Savran, D. (eds.) (2002), The Masculinity Studies Reader, Oxford, WileyBlackwell.

Barker, J. (2008), 'A Hero Will Rise': The Myth of the Fascist Man in Fight Club and Gladiator.” Literature-Film Quarterly 36 (3), pp. 171-187.

Berghaus, G. (1996), Futurism and Politics: Between Anarchist Rebellion and Fascist Reaction, 1909-1944, Providence, RI, Berghahn Books.

Carrigan, T., Connell, B. \& Lee, J. (1985), Toward a New Sociology of Madculinity, Theory and Society, 14(5), pp. 551-604.

Connell, R. W. (1995), Masculinities, Sydney, Allenn \& Unwin.

Cuordileone, K.A. (2005), Manhood and American Political Culture in the Cold War, New York \& London, Routledge.

Jarillot Rodal, C. (2010), Manifiesto y vanguardia. Los manifiestos del futurismo italiano, dadá y el surrealismo, Bilbao, Servicio Editorial de la Universidad del País Vasco.

Fincher, D. (1999), Fight Club, Fox 2000 Pictures.

Giroux, H. A. (2003), Cine y entretenimiento Elementos para una crítica política del filme. Barcelona, Paidós. 
Gronstad, A. (2003), One-Dimensional Men: Fight Club and the Poetics of the Body. Film Criticism. 28(1), pp. 1-23.

Kimmel, M. (1996), Manhood in America: A Cultural History, New York, Free Press.

Ling, P. \& Monteith, S. (eds.) (1999), Gender in the Civil Rights Movement, New York \& London, Garland Publishing Inc.

Marinetti, T. F. (1968), Manifiestos y textos futuristas, Barcelona, Ediciones del Cotal.

Mead, M. (1935), Sex and Temperament in three primitive societies, New York, William Morrow and Company.

Mosse, G. (1996), The Image of Man. The Creation of Modern Masculinity,. Oxford \& New York, Oxford University Press.

Orgeron, D. (2002), David Fincher, en: Y. Tasker (ed.): Fifty Contemporary Filmmakers, London \& New York, Routledge, pp. 154-160.

Reeser, T. W. (2010), Masculinities in Theory: An Introduction, Chichester, Wiley-Blackwell, Oxford University Press.

Ward, L. F. (1883), Dynamic Sociology, New York, Appleton.

Whitehead, S. M. \& Barret, F. J. (eds.) (2001), The Masculinities Reader, Cambridge, UK $\&$ Malden, MA, Polity Press. 